\title{
Study of n-on-p sensors breakdown in presence of dielectrics placed on top surface
}

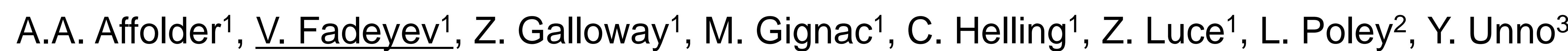
${ }^{1}$ University of California Santa Cruz, USA, ${ }^{2}$ Deutsches Elektronen-Synchrotron, Hamburg, Germany, ${ }^{3} I P N S$, KEK, Tsukuba, Ibaraki, Japan

\section{ABSTRACT}

The ATLAS Experiment at LHC will have several upgrade projects for High Luminosity LHC operations. Its tracking system will be replaced to cope with the higher interaction rate and radiation levels. The Strip portion of the tracker will be significantly expanded in radius and instrumented area to control the occupancy and momentum resolution. The strip modules are based on large-area n-on-p sensors with short strips, designed to work with the larger particle fluxes and radiation hardness requirements. The strip module design has readout flex circuit glued directly on top of the sensors' active area to facilitate the assembly process and minimize the radiation length. Adhesive spread outward to the guard ring (GR) region is typically avoided to control the sensor breakdown. However, due to the large number of modules to be constructed, on the order of 20000 , such occasions may in principle happen, depending on the process precision control. Therefore, the adhesive influence on the sensor breakdown and the breakdown mechanism are of interest.

In this contribution we report on the studies of the breakdown behavior with prototype sensors, where adhesives were placed on top of the sensor, either directly in the GR region, or in the active area far away from it. Several adhesives under consideration for module building were used in these measurements. The measurements after thermo-cycling and after irradiation were also performed.

\section{INTRODUCTION}

We have performed two separate studies to check compatibility of a variety of glues with the prototype n-on-p sensors.

Study A (more details can be found in [1]):

o Glues: Epolite FH-5313, Loctite 3525, DYMAX 3013, DYMAX 6-621

o Sensors: ATLAS07 [2]

o Placement: active area

o Irradiation: yes

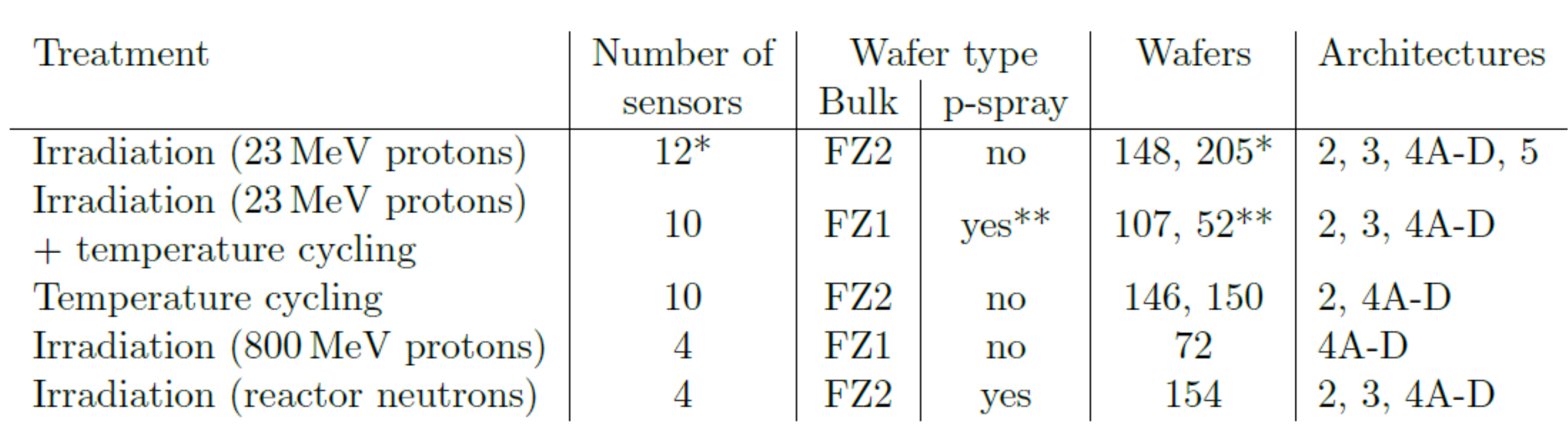

Study B:

Table 1 Sensor types, their properties, and test procedures used in Study A (from [1])

o Glues: Epolite FH-5313, Polaris PF 7006, Henkel F112

o Sensors: ATLAS07 [2] and ATLAS12 [3]

o Placement: active area, GR region, edge of the sensor

o Irradiation: not yet

\section{STUDY A}

In the study $A$ the glues were deposited on top of active area of the sensors avoiding the proximity to the GR region. The deposition was manual, with a pipette, however the placement precision was adequate for this purpose. The examples of the deposition are placement precision was adequate for this purpose. The examp
shown in Figure 1. More details are available in reference [1].

Two leakage current assessments before and after deposition showed a slight increase of the currents and decrease of the breakdown voltages (Figure 2).

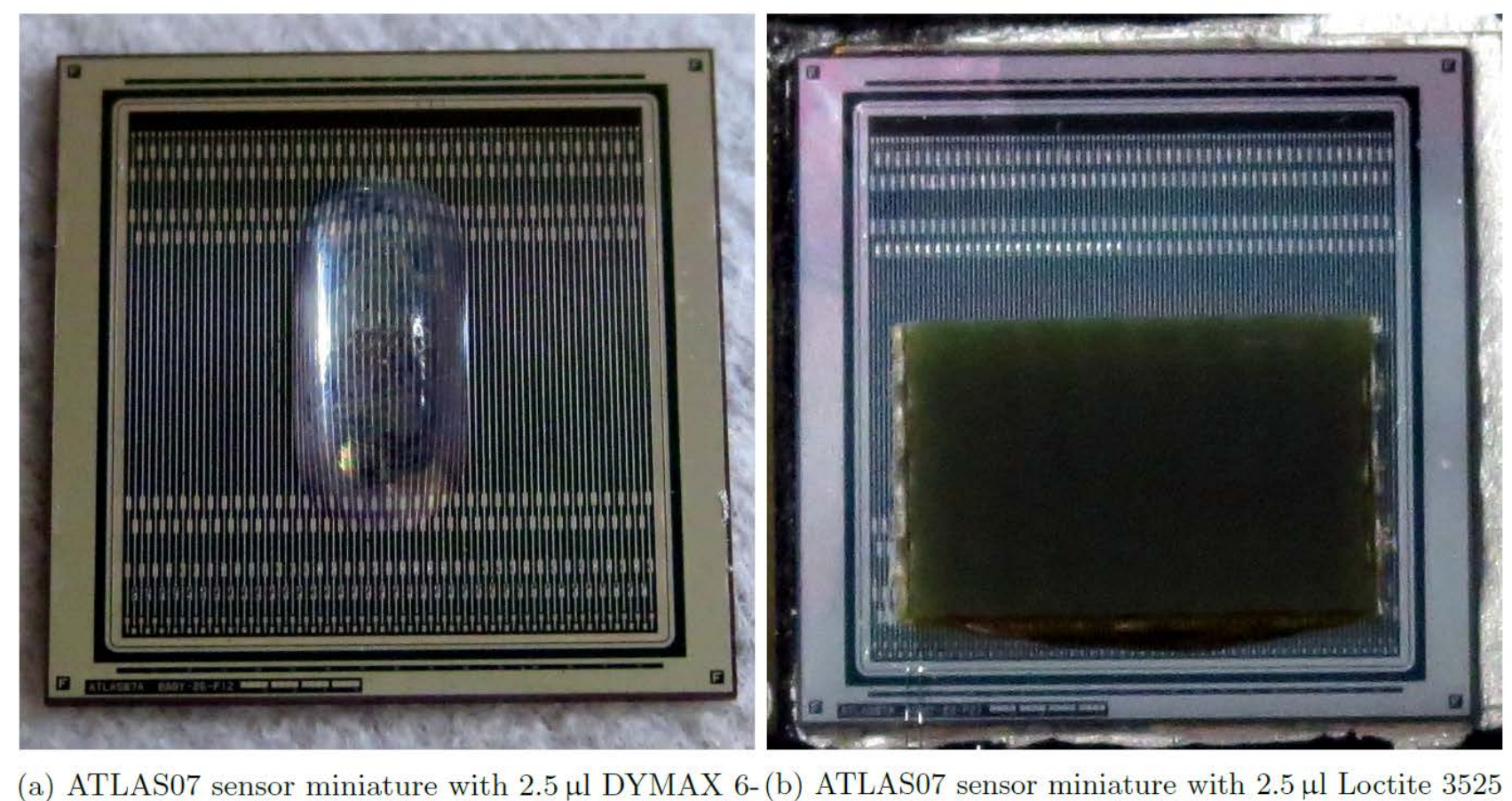

Figure 1 Examples of the glue deposition on top of prototype mini-sensors used in Study A.
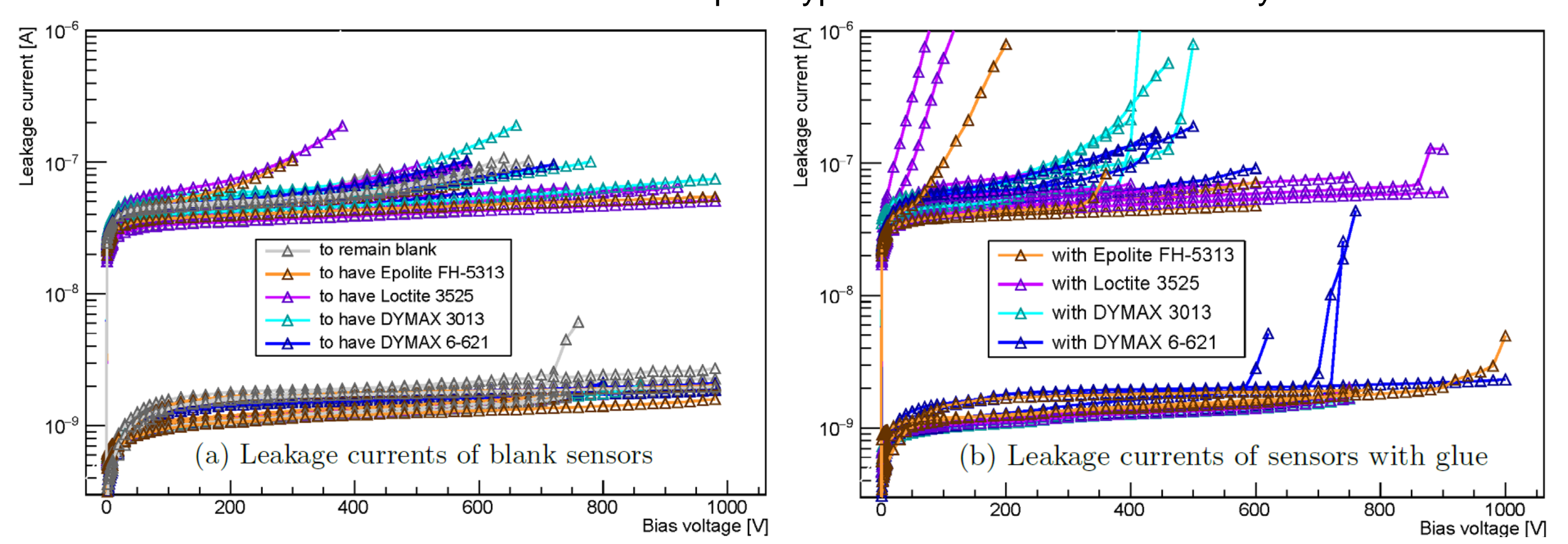

Figure 2 IV characteristics of mini-sensol

Some of the samples in the study were subjected to thermo-cycling: 100 repetitions of 6 hours at $-20 \mathrm{C}$ and 6 hours at $50 \mathrm{C}$, with 1 hour ramp in-between. The process alleviated some of the early breakdown features (Figure 3). This could be due to the change in glue characteristics, e.g. attenuation of the mechanical stress caused by the glue. The results did not depend on the glue type in this test.

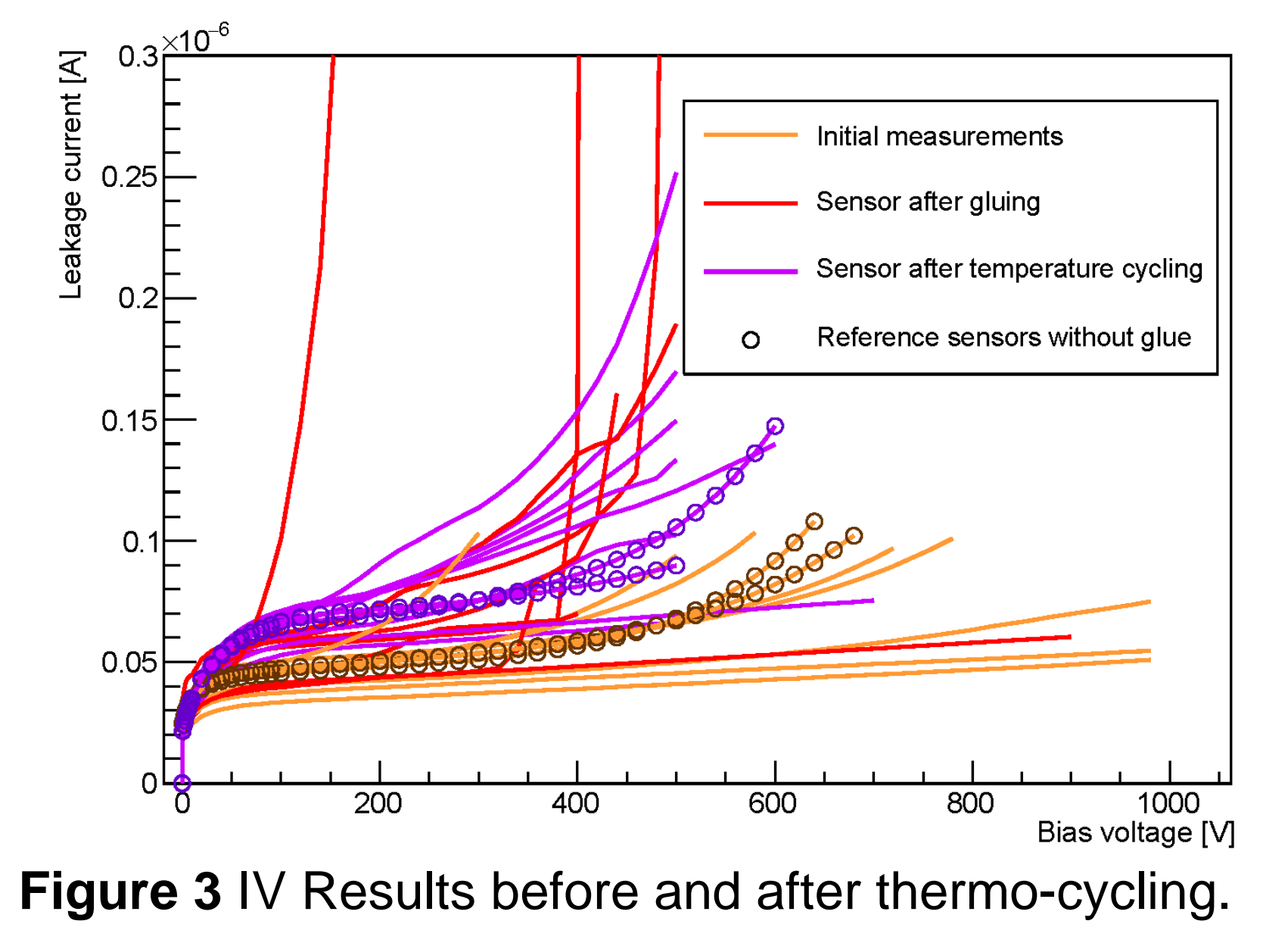

\section{References}

1) L. Poley, "Studies of adhesives and metal contacts on silicon strip sensors for the ATLAS Inner Tracker". PhD Thesis, Humboldt University

2) $Y$. Unno et al, "Development of n-on-p silicon sensors for very high radiation Y.

3) $Y$. Unno et al, "Development of $n^{+}$-in-p large-area silicon microstrip sensors for very high radiation environments - ATLAS12 design and initial results", NIM A 765 (2014) 80-90

\section{SCIPP} FOr PARTICLE PHYSICS

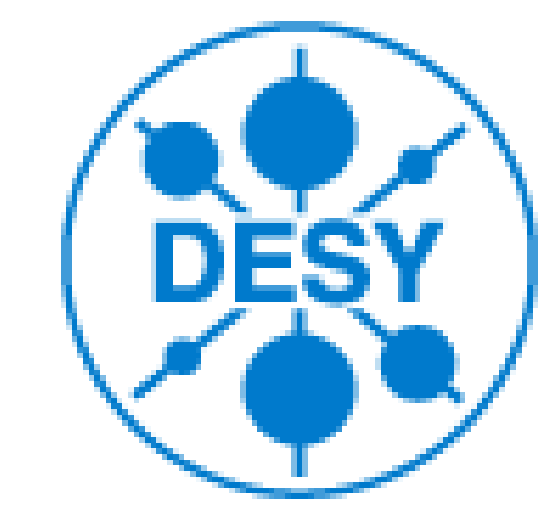

STUDY A: IRRADIATION

Some of the sensor-glue samples used in Study A were irradiated with protons at KIT and Los Alamos, as well as with neutrons in Ljubljana. The irradiation fluence was $2 \times 10^{15} \mathrm{neq} / \mathrm{cm}^{2}$ in al cases. A typical post-irradiation performance is shown in Figure 4. In all cases there was an expected increase of leakage current, but no sign of early breakdowns or obvious glue type dependence.



Figure 4 IV characteristics of sensor samples with glues after irradiation with $23 \mathrm{MeV}$ protons at KIT.

\section{STUDY B}

In Study B initially the glue drops were placed on top of GR region, to find out if this is a dangerous location for the glue (Figure 5).

The study of the breakdown behaviour in this case showed a strong reduction of the breakdown voltage in most cases (Figure 6). The effect did not depend on thermo-cycling (1 hour at 50 deg C). However, we suspected that this could be due to the glue breakdown under HV. The glue placement created a possible path for electric discharge from $\mathrm{HV}$ present on the sensor edge to a passivation opening on the bias ring (Figure 7)
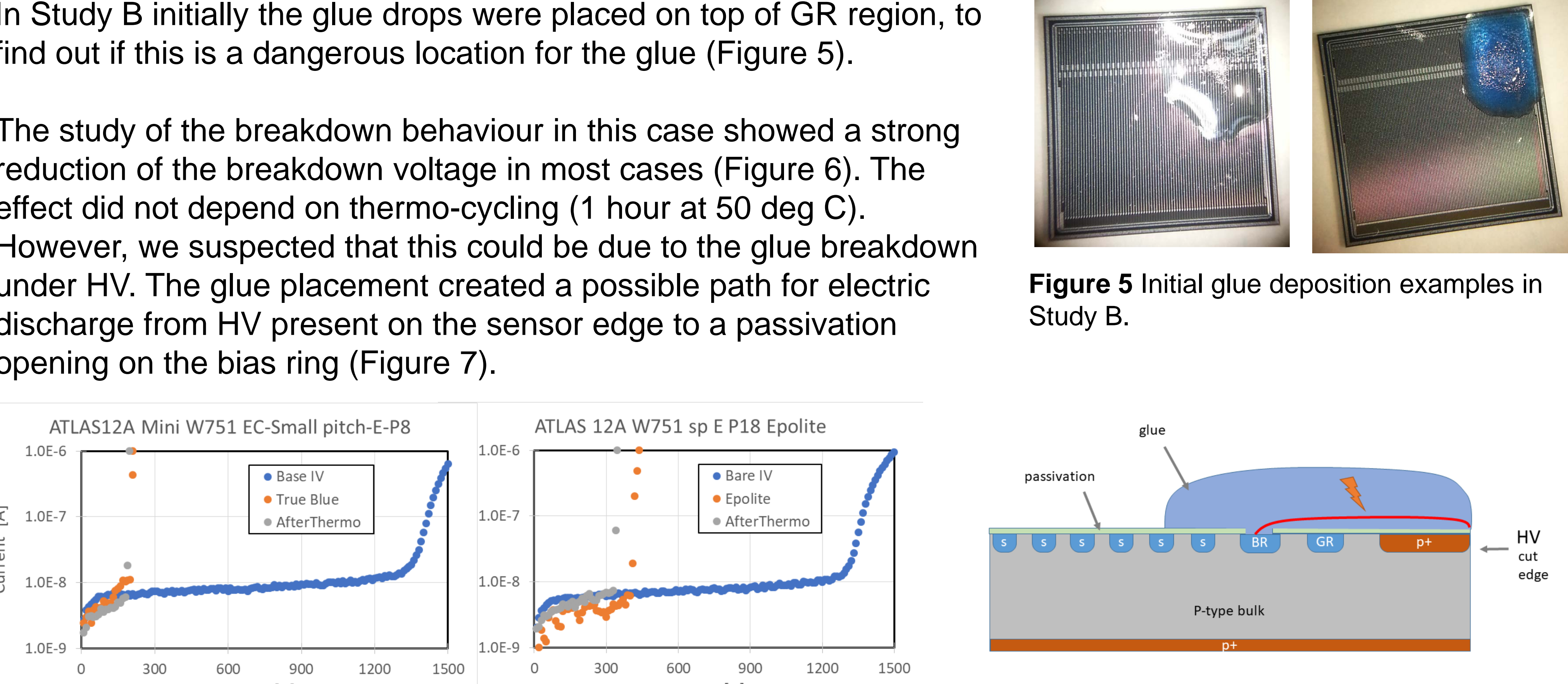

Figure 5 Initial glue deposition examples in Study B.

with placement indicated in Figure 5 .

We then approached the issue of the glue placement in a more systematic way (Figure 8). Different placements were targeted: $G R$ active region, proximity to the sensor edge. differentiate the dependence of the sensor breakdown on the glue placement from the humidity effects, data were taken at both ambient humidity and dry atmosphere in al cases.

The results at different stages of the glue placement are shown in Figure 9. In some cases the ambient humidity affected the breakdown voltage, however this artifact was removed when testing in dry atmosphere. Only the "edge" placement of the glue resulted in a significant and permanent reduction of the breakdown voltage.

The method was also applied to a full-sized ATLAS07 sensor, with similar results (Figure 10)
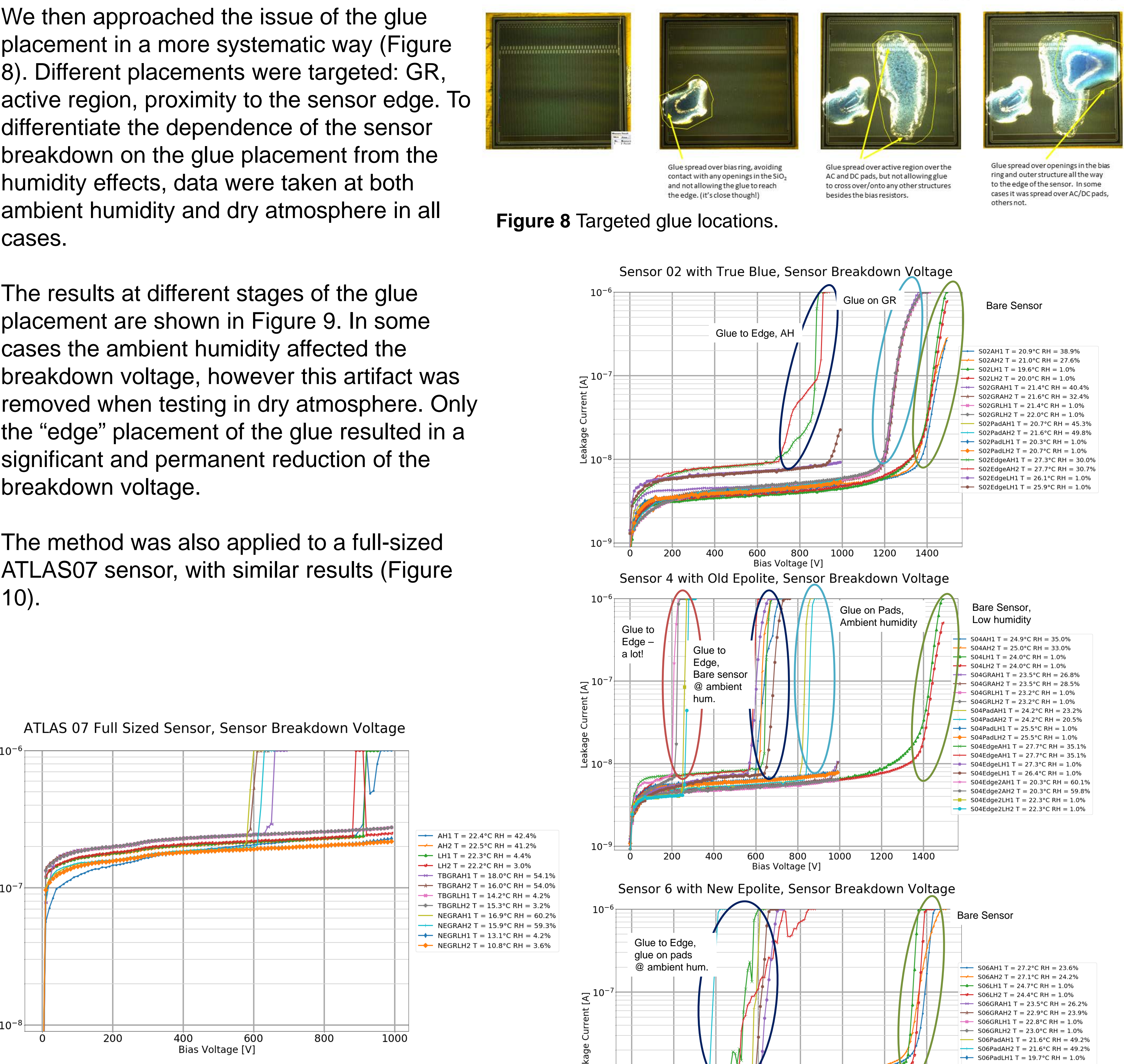

Figure 8 Targeted glue locations.
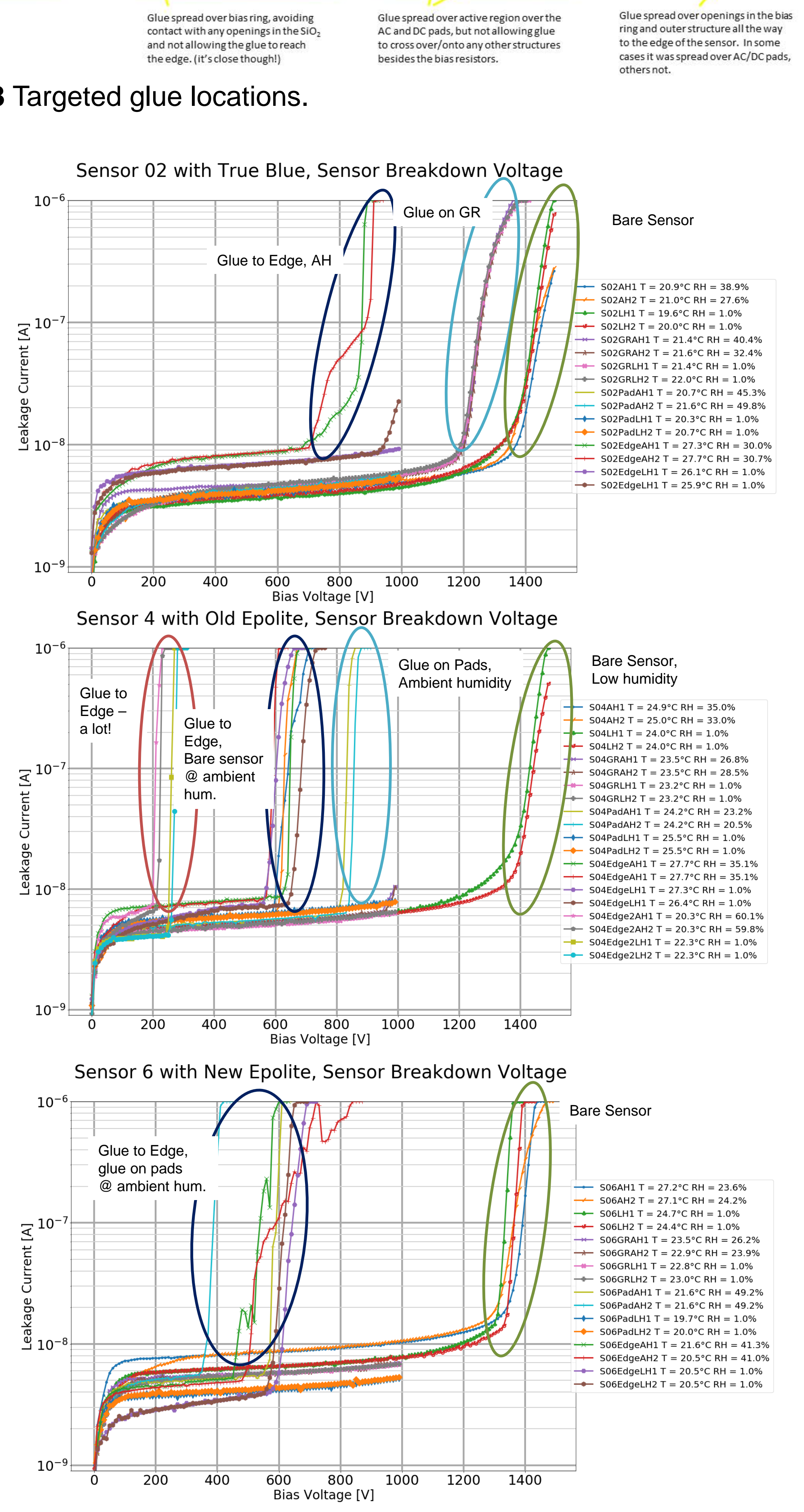

Figure 9 IV characteristics of ATLAS12 mini-sensors with different glues for different placement locations and humidity.

CONCLUSIONS

We have performed sensor breakdown studies in presence of a variety of glues placed on top surface at different locations. There are several conclusions we can draw from the results:

The glue placement on top of active region generally does not affect the sensor breakdown in a significant way. In some cases we saw earlier breakdowns that could either be "cured" by thermo-cycling, or were clearly due to sensor sensitivity to humidity. In the latter case tests at low humidity resulted in $\mathrm{V}(\mathrm{bd})>1000 \mathrm{~V}$.

- The breakdown after irradiation was not seen, and the glue effect was not significant.

o The glue placement on top of guard ring region did not cause the early breakdown (except at high humidity).

o Glue placement close to the sensor edge is indeed dangerous, due to possible electric path connecting HV on the edge with exposed pads at ground potential (e.g. DC contacts or bias pads). 